\title{
Religions and Global Sustainability. A research study in Catalonia (Spain)
}

\author{
Silvia Albareda-Tiana, Montserrat Gas-Aixendri ${ }^{1}$
}

\begin{abstract}
There is very little scientific literature that considers the relationship between religions and an integral concept of Sustainable Development, including its three dimensions (social, economic and environmental), as proposed in the UN Sustainable Development Goals. It was necessary to conduct a study of this kind, with the aim of making visible and show with concrete data, the contribution of religions to the integral sustainability.

This paper presents an empirical research in which we intend to make visible the actions performed by religious organizations in Catalonia, that have been contributing to the Sustainable Development. The study is the launching of the Sustainability and Religions Watch.

This study about the contribution of religious organizations to the Sustainable Development has been made by a mixed method with two techniques for data collection: firstly, has counted the presence of indicators linked to the priority areas of Sustainable Development, from the analysis of the content of the websites of religious organizations (quantitative analysis). Moreover, semistructured in-depth interviews were conducted to representatives of religious organizations (qualitative analysis).

The study of the contribution of religions to the Sustainable Development opens new doors to interfaith dialogue because it is based on elements that are common between different beliefs. This dialogue is a path that can effectively contribute to peace.
\end{abstract}

Key words: Sustainability and Religions; Interfaith dialogue; religions and social peace.

\section{Il contesto della ricerca}

\subsection{Introduzione}

La contribuzione delle religioni allo sviluppo sostenibile non è nuova. Nel 2015 ha avuto una portata senza precedenti nel pubblicarsi l'enciclica Laudato si' di Papa Francesco e la Interfaith Declaration on Climate Change(IDCC), prima del Vertice sul Cambiamento Climatico COP21 a Parigi. La Dichiarazione raccoglie i principi condivisi da tutte le religioni del mondo e dà fondamento al contributo delle religioni allo sviluppo sostenibile (IDCC, 2015). Questa responsabilità delle religioni verso la cura del pianeta e dei suoi abitanti non si è manifestata solo nel terreno teorico e delle dichiarazioni d'intenzioni, ma si è tradotto spesso in azioni concrete che contribuiscono alla sostenibilità integrale. Malgrado tutto, questo contributo è ancora poco visibile.

Secondo gli ultimi informi globali in materia di coesione sociale e sostenibilità il contesto mondiale odierno non ha precedenti. Rapporti scientifici come il Climate change: Synthesis Report (IPCC, 2014) raccolgono prove scientifiche sull'aumento della temperatura che sta producendo il cambiamento climatico, con gravi conseguenze 
economiche e sociali per le popolazioni umane e gli ecosistemi. I rapporti elaborati dal Fondo Mondiale per la Natura (WWF, 2012 e 2014) rendono palese il fatto che consumiamo beni e produciamo residui ad un ritmo molto più accelerato di quanto la Terra possa generare e supportare. Dalla Rivoluzione Industriale ad oggi sono aumentati i livelli di $\mathrm{CO}_{2}$ atmosferico e fin dagli anni sessanta del secolo scorso ci sono problemi d'inquinamento e di esaurimento delle risorse naturali. Nel XXI secolo questi problemi si sono globalizzati e hanno acquistato dimensioni planetarie.

Di fronte a queste situazioni i leader religiosi hanno firmato diverse dichiarazioni chiedendo il rispetto del pianeta, la mitigazione del cambiamento climatico e la pace a livello mondiale. Queste attuazioni non sono state visibili, mentre i mass media negli anni 2014 e 2015 hanno diffuso notizie ed immagini su atti di terrorismo presuntamente religioso. Spesso si pensa alle religioni come elementi di conflitto, di confrontazione sociale e anche come fenomeni legati alla violenza (nel caso specifico di alcuni gruppi fondamentalisti).

In questo articolo intendiamo presentare un modello pratico (concretamente un Osservatorio avviato sulla base di una ricerca empirica) per far diventare più visibili le azioni delle entità religiose in favore dello sviluppo sostenibile. L'Osservatorio ha una base territoriale limitata: la Catalogna (Spagna). Siamo convinti che i risultati sono perfettamente estrapolabili ad altri luoghi, regioni e paesi. Si prendono come punto di partenza le diverse dimensioni dello sviluppo sostenibile e si mettono in rapporto con le attività realizzate per le entità religiose in modo che si possa quantificare in qualche misura il contributo delle religioni alla sostenibilità.

$\mathrm{Si}$ tiene anche conto delle convergenze esistenti tra le diverse religioni nei confronti dello sviluppo sostenibile quale scopo comune. In questo senso l'Osservatorio che presentiamo vuole anche contribuire al dialogo interreligioso e alla pace. Lo studio delle convergenze tra religioni in ciascuno degli ambiti prioritari dello sviluppo sostenibile (ad esempio la promozione dei diritti umani, la riduzione della povertà o l'educazione, tra gli altri) apre nuovi orizzonti per il dialogo interreligioso poiché si fonda in elementi che sono comuni a tutte le persone indipendentemente dalle loro credenze. Questo dialogo è un cammino che può contribuire in modo efficace allo sviluppo umano e alla pace delle nostre società.

\subsection{Cornice teorica}

Una parte importante della letteratura scientifica internazionale che si è occupata del rapporto tra religione e sostenibilità si è focalizzata nello studio del rapporto tra religioni e ambiente (Boyd, 1999; Gottlieb, 2006; Grim\&Tucker, 2014; Djupe, 2010; Hunt, 1999; Sherkat, 2007; Reuter, 2015; Tucker, 2008 e White, 1967) e anche nel rapporto tra religioni e cambiamento climatico (Brown, 2013 e Wolf, 2009). Si è anche studiato come le convinzioni religiose hanno inspirato questo tipo di attivismo ambientale. Altri lavori si sono incentrati nel rapporto tra religioni e sviluppo con riferimento agli aspetti sociali ed economici nei paesi in via di sviluppo. Per contrasto c'è poca letteratura che consideri il rapporto tra religioni e la sostenibilità in senso integrale, cioè includendo le dimensioni sociali, economiche e ambientali, così come viene proposto dall'ONU nell'Agenda 2030 (ONU, 2015). Autori come Berry (2014), Minton(2015) e Narayanan(2014) coincidono nel sottolineare il contributo delle religioni 
allò sviluppo sostenibile e in particolare alla mitigazione del cambiamento climatico. Gli studi pubblicati fino al momento a livello internazionale sono riflessioni teoriche di tipo umanistico od interpretativo. Si sono realizzati pochi studi empirici sul contributo degli enti religiosi allo sviluppo sostenibile. Un precedente immediato della nostra linea di ricerca è lo studio di Aguilar e Coscolla (2014) dove si mostra il contributo della Chiesa cattolica alla coesione sociale nella Catalogna.

\subsection{Le religioni e lo sviluppo sostenibile}

Il termine "sostenibilità" e l'espressione "sviluppo sostenibile" sono utilizzati con frequenza, ma spesso non si comprende il suo significato globale oppure viene associato solo agli aspetti ambientali (riciclaggio di residui, efficienza energetica, ecc.) senza riconoscere le dimensioni sociali ed economiche che le sono proprie. All'interno del Rapporto Brundlant viene definito lo sviluppo sostenibile come quello sviluppo che tiene conto delle necessità della generazione presente senza mettere a rischio i bisogni delle generazioni future (Brundtland, 1987).

Si propone così un'approccio allo sviluppo umano globale e intergenerazionale il quale comprende una visione integrale della sostenibilità nella sua triplice dimensione: economica (includendo la riduzione della povertà e l'incremento dell'equità tra paesi e regioni), sociale (coesione sociale e promozione dei diritti umani)e ambientale o ecologica (conservazione delle risorse naturali e mitigazione del cambiamento climatico). Questo concetto integrativo della sostenibilità viene riconosciuto in modo esplicito tra gli Obiettivi legati allo Sviluppo Sostenibile (Sustainable Development Goals) dell'ONU per i prossimi quindici anni: Trasformare il nostro mondo: l'Agenda 2030 per lo Sviluppo Sostenibile (ONU, 2015).

Negli ultimi decenni sono state celebrate importanti riunioni a livello internazionale sul contributo delle religioni alla sostenibilità. Sono anche stati pubblicati documenti sui valori e le credenze in cui poggiano la riflessione e l'azione per promuovere la sostenibilità (ACT, 2015; ARC, 1986). Diverse confessioni religiose hanno promosso atti e firmato documenti d'impegno per la protezione del pianeta. Il mese di giugno dell'anno 2015 Papa Francesco ha pubblicato l'Enciclica Laudato si', la quale costituisce un appello alla cura del creato e di ogni essere vivente, facendo un uso responsabile e solidale delle risorse. Francesco parla sull'intimo rapporto tra le situazioni di povertà e la fragilità del pianeta. Nel documento si presenta una nuova visione dello sviluppo mondiale, più integrale. Si mette in rapporto il senso umano dell'ecologia con la cura dell'ambiente e delle persone e si richiama ad altre forme per capire l'economia ed il progresso (Francesco, 2015b). L'Enciclica ha avuto in poco tempo ripercussione a livello internazionale ed i suoi contributi sono stati valutati molto positivamente da personalità rilevanti in ambiti molto diversi, come ad esempio il Segretario Generale dell'ONU Ban Ki-moon (ONU, 2015a).

Con lo scopo di offrire un contributo alla COP21, lo scorso 21 ottobre 154 rappresentanti di diverse confessioni religiose hanno sottoscritto nella sede di Nazioni Unite una Dichiarazione Congiunta sul Cambiamento Climatico. Nel testo della Dichiarazione si sottolinea il fatto che tutte le religioni del mondo si basano sui principi del rispetto e reverenza alla vita e al creato. Si constata che la causa del cambiamento climatico è dovuta all'agire dell'uomo e si riconosce la responsabilità etica di tutti, dalla quale 
dipende il futuro dell'intera umanità. Il documento dichiara l'impegno di tutte le religioni nella promozione del cambiamento nei comportamenti delle persone. Oltre a questa Dichiarazione interreligiosa alcune confessioni come quella cattolica, islamica e buddista hanno reso pubbliche le loro proprie dichiarazioni ${ }^{1}$.

\subsection{Il contesto religioso della Catalogna}

Per rendere visibile il contributo delle religioni allo sviluppo sostenibile nella Catalogna il punto di partenza è stato conoscere quale confessioni religiose operano nel territorio. A questo scopo è stato richiesto l'elenco ufficiale alla Direcció General d'Afers Religiosos (Departament de Governació i Relacions Institucionals) della Generalitat, Governo autonomico della Catalogna. Secondo questi dati troviamo un totale di 12 confessioni religiose. La religione più antica e più radicata è quella cattolica. La presenza di questa Chiesa alla Catalogna risale al I secolo. È tuttora la religione con un numero più elevato di fedeli (secondo dati del 2014 più del 60\% dei cittadini si riconoscono come tali, anche se i battezzati sono di più). Allo stesso tempo si è prodotto un incremento della popolazione appartenente ad altre denominazioni religiose che sono minoritarie (circa un 5,7\% della popolazione). L'effetto della secolarizzazione si manifesta in un incremento degli agnostici e degli atei (circa il $32 \%$ della popolazione) (DGAR, 2014).

Ci sono dati sulla presenza di fedeli protestanti nella Catalogna fin dal XVI secolo (González \& Correa, 2014, p. 24). Le prime comunità stabili risalgono però al XIX secolo. D'accordo con i dati ufficiali sono radicate in questo territorio le seguenti denominazioni protestanti: Anglicani, Assemblee di Fratelli, Assemblee deiFratelli Darbista, Battisti, Luterani, Mennoniti, Metodisti, Neotestamentari, Pentecostali, Presbiteriani, Quaccheri, Unitari, una aggregazione interdenominazionale e una di comunità non determinate (DGAR, 2014).

La percentuale di popolazione straniera nella Catalogna è cresciuta negli ultimi decenni come conseguenza dei flussi migratori. Questo ha avuto delle precise conseguenze nel panorama della diversità religiosa. Secondo i dati dell'Osservatorio del Pluralismo Religioso nel 2014 i fedeli musulmani nella Catalogna erano 509.333. Si tratta cioè della comunità autonoma spagnola con maggior numero di membri di questa confessione. Nell'insieme della Spagna i musulmani sono 1.850 .000 circa, secondo lo Studio Demografico della Popolazione Musulmana elaborato dall'Unione di Comunità Islamiche della Spagna (UCIDE) e l'Osservatorio Andalusí. Il 60\% de la popolazione islamica spagnola sono stranieri. Esiste anche un nucleo di conversi all'Islam sopratutto per motivi di matrimonio (Manzano, 2013).

Le altre comunità religiose presenti nella regione catalana sono le Chiese orientali (ortodossa e copta), le Comunità ebraiche, gli Avventisti, i Mormoni, i Testimoni di Geova, la Comunità Baha'í, gli Induisti, i Sikh, i Buddisti ed i Taoisti. La presenza di queste comunità religiose ha origini recenti (seconda metà del XX secolo) e la grande maggioranza proviene dell'immigrazione. Questa realtà condiziona il fatto che molte di queste comunità concentrano i loro sforzi nell'aiuto materiale e nell'integrazione dei loro

1 Nel sito web dell'OsservatoriosuSostenibilità e Religioni si possonotrovare i documenti citati lungo i lavoro insieme ad altre risorse: http://www.sustainabilityandreligions.org/documents/[consultata a maggio 2016]. 
fedeli alla società catalana (cercando alloggio, alimenti e aiuto per cercare lavoro) e in attività destinate a rafforzare le loro credenze in un contesto nel quale sono in minoranza. Non avendo un forte radicamento, molte volte non hanno ancora la capacità (sociale ed economica) di prestare aiuto ad altre persone al di fuori delle loro comunità di fedeli.

\section{Descrizione}

\subsection{Studio antecedente}

L’Osservatorio su Sostenibilità e Religioni pretende di rendere "visibili" i contributi "invisibili" delle entità religiose alla sostenibilità della società. Il primo passo che si è dovuto fare è conoscere di prima mano quali sono questi contributi. È stato così realizzato uno studio esploratorio sulle confessioni religiose impiantate nella Catalogna e sulle loro attività collegate alla sostenibilità. Si è cercato di capire in quale misura queste entità realizzano un contributo specifico allo sviluppo sostenibile della società catalana. Questo lavoro è stato realizzato mediante uno studio empirico che segue una metodologia mista (quantitativa e qualitativa). Per l'esplorazione quantitativa abbiamo preso come base l'elenco ufficiale di enti religiosi proporzionato Direzione Generale d'Afferi Religiosi del Governo autonomo della Catalogna (Generalitat de Catalunya). I dati sulle attività e servizi promossi dalle entità religiose è stata fatta sulla base dei contenuti dei siti web delle organizzazioni ${ }^{2}$. Sono state selezionate e classificate solo quelle attività collegate allo sviluppo sostenibile. I criteri per scegliere le attività e per classificarle sono quelli che l'UNESCO ha stabilito nel $2005^{3}$ e che sono evidenziati in seguito sulla Tavola 1 .

Sono stati analizzati i siti Internet e facebook delle entità religiose che riguardano il banco dati ceduto dalla Direzione Generale di Affari Religiosi della Generalitat. Abbiamo collegato le diverse attività e servizi con i settori prioritari dello sviluppo sostenibile e abbiamo identificato la tipologia delle attività con i diversi item appartenenti a ciascun settore. Ciascuna attività è stata legata ad uno solo degli item. Siamo consci che questa raccolta di dati è povera e presenta limiti importanti, ma riflette comunque una parte di una realtà che è più ricca e complessa.

2 La parte quantitativa della ricerca ha avuto una limitazione importante per quanto riguarda la Chiesa cattolica poiché il numero di organizzazioni cattoliche era troppo grande. Così abbiamo preso come punto di partenza i risultati di uno studio previo fatto nell'Università Rovira i Virgili dal Prof. A. Belzuncegui nel 2014 e dal quale risultano circa 250 entità cattoliche attive nell'ambito sociale.

3 Abbiamo utilizzato come riferimento un documento pubblicato nel 2005 dall'Organizzazione delle Nazione Unite per l'Educazione, la Scienza e la Cultura all'inizio della Decada dell'Educazione per lo Sviluppo Sostenibile e che citeremo come UNESCO 2005. Questiaspetti prioritari sono coincidenti nella prattica con gli Obiettivi per lo Sviluppo Sostenibile (Sustainable Development Goals) approvati dall'ONU a settembre 2015. Questi obiettivi non erano ancora stati formulati e pubblicati all'inizio della ricerca. Per questo motivo abbiamo utilizzato come documento di riferimento UNESCO, 2005. 
Tavola 1. Settori prioritari dello sviluppo sostenibile (Fonte: UNESCO, 2005: 5)

\begin{tabular}{lll}
\hline Dimensione Socioculturale & $\begin{array}{l}\text { Dimensione } \\
\text { Mediambientale }\end{array}$ & $\begin{array}{l}\text { Dimensione } \\
\text { Economica }\end{array}$ \\
\hline Diritti umani & Risorse naturali & Povertà \\
$\begin{array}{l}\text { Diversità culturale ed } \\
\text { Educazione }\end{array}$ & $\begin{array}{l}\text { Mitigazione del cambiamento } \\
\text { climatico }\end{array}$ & $\begin{array}{l}\text { Responsabilità sociale } \\
\text { corporativa }\end{array}$ \\
Pace e sicurezza umana & Trasformazione rurale & Economia sostenibile \\
Eguaglianza & Urbanismo sostenibile & \\
Salute & Problematica ambientale & \\
Governance & & \\
\hline
\end{tabular}

Per poter completare le limitazioni di questa raccolta parziale di dati quantitativi e anche con l'intenzione di conoscere quale è la percezione del contributo allo sviluppo sostenibile da parte delle stesse confessioni religiose nella Catalogna, abbiamo realizzato interviste in profondità (interviste semi strutturate focalizzate) a rappresentanti autorevoli delle 12 confessioni religiose che operano nel territorio. Queste interviste sono state processate ed analizzate con il metodo della ricerca qualitativa e l'aiuto del programma Atlas-ti. Questa parte del lavoro si è basato nell'analisi e codificazione di un totale di 18 interviste realizzate ad "informanti-chiave" che rappresentano le religioni con presenza nella Cataloga. La distribuzione delle interviste è stata questa: Chiesa cattolica (tre rappresentanti: Delegazione Pastorale dell'arcidiocesi di Barcellona, Caritas ed Unione dei Religiosi della Catalogna); Chiese protestanti; Avventisti; Chiese ortodosse; Chiesa Copta; Comunità musulmane (due rappresentanti: Comunitat Musulmana de Catalunyae Consell Islàmic de Catalunya); Comunità ebraiche; Mormoni; Testimoni di Geova; Fede Bahá'i; Induismo (Due rappresentanti: Induismo Advaita e Hare Krishna); Sikh; Buddisti e Taoisti.

Il contenuto delle interviste è stato registrato e trascritto per essere poi sottoposto ad un processo di analisi concettuale mediante il programma Atlas.ti, il quale permette la codificazione delle risposte e serve a dare un contenuto quantitativo alle medesime. La codificazione consente classificare e raggruppare risposte secondo categorie. Codificare significa creare categorie e metterle in rapporto con il contenito delle risposte in un modo razionale, ordinato, semplificato e quantificabile. I nostri codici sono stati appunto gli item dei settori dello sviluppo sostenibile d'accordo al documento UNESCO (2005)(Tavola 1). Atlas.ti ci consente finalmente di creare, a partire da questa analisi, dei mappi concettuali ove è possibile contabilizzare il numero di volte che è stato fatto riferimento a ciascuno degli item dello sviluppo sostenibile nelle interviste ai leader religiosi.

\subsection{Creazione del sito web e futuri sviluppi}

La prima applicazione collegata all'avvio dell'Osservatorio su Religioni e Sostenibilità è la pubblicazione del sito web www.sustainabilityandreligions.org_per rendere visibile il contributo delle religioni allo sviluppo sostenibile. Il sito web creatosi 
include e rende pubblici i risultati di una prima esplorazione realizzata sulla base dell'informazione disponibile su Internet. Nei nostri giorni Internet è una delle principali fonti d'informazione e per tanto il luogo adatto per potenziare la visibilità delle persone e degli enti che agiscono pubblicamente.

Il sito web contiene anche documenti e riferimenti bibliografici sul rapporto tra religioni e sostenibilità. Così, l'Osservatorio vuole diventare uno dei punti di riferimento per coloro che vogliono conoscere e studiare meglio questa tematica con le implicazioni che comporta (vid. Conclusioni ed implicazioni).

La seconda fase del lavoro prevede l'incorporazione di altra informazione ed il collegamento del sito web dell'Osservatorio con i siti web degli enti religiosi che realizzano attività collegate con la sostenibilità. L'Osservatorio prevede la possibilità di organizzare attività di formazione e sensibilizzazione sul contributo delle religioni alla sostenibilità integrale.

\section{Risultati}

L'esplorazione quantitativa realizzata ha considerato la contribuzione delle 12 confessioni religiose presenti alla Catalogna al suo sviluppo sostenibile. Sono state analizzate i siti web di circa 500 entità religiose. Tutte le confessioni religiose realizzano azioni collegate alla dimensione sociale della sostenibilità come ad esempio: la promozione dei diritti umani, l'educazione, la promozione della sicurezza, la pace ed il dialogo, tra altre. Sono state conteggiate circa 2000 attività vincolate con la dimensione sociale e culturale della sostenibilità. Per quanto riguarda la dimensione economica dello sviluppo sostenibile le entità cattoliche, protestanti, ebraiche e la Fede Baha'í, realizzano contribuzioni effettive per attenuare le situazioni di povertà estrema con aiuto per l'alimentazione, il vestiario e l'abitazione. Altre religioni minoritarie promuovono iniziative sociali ma in altri paesi in vie di sviluppo, non nella Catalogna. Abbiamo conteggiato 500 attività collegate alla dimensione economica della sostenibilità. Infine, non abbiamo trovato nessuna attività con riferimento alla sostenibilità ambientale: non sono state individuate iniziative vincolate con l'uso sostenibile delle risorse naturali oppure diretti a frenare il cambiamento climatico e le sue conseguenze. I contributi delle entità religiose vincolate a questa dimensione della sostenibilità non sono visibili. La parte qualitativa della ricerca ha rivelato che esiste invece una preoccupazione reale ed effettiva in tutte le religioni per quanto riguarda la conservazione e la cura del pianeta. Ma questa preoccupazione no si manifesta ancora nella promozione di attività concrete.

Le interviste realizzate ai rappresentanti delle confessioni manifestano l'interesse e la preoccupazione per la cura dell'ambiente. Nelle religioni monoteiste (cristiani, ebrei e musulmani) esiste una visione creazionista del mondo che invita ad avere un atteggiamento di cura del mondo e di ringraziamento alla divinità per averlo fatto, e promuovono un uso responsabile delle risorse naturali. In altre spiritualità d'origine orientale (induisti, sikh, buddisti e taoisti) si percepisce un grande rispetto verso la natura che viene considerata in certo modo come qualcosa di sacro. Queste comunità religiose hanno anche un senso molto chiaro dell'interdipendenza tra tutti i viventi.

Riassumendo, tutte le religioni nella Catalogna manifestano ragioni profonde per promuovere la cura dell'ambiente. Ma nella pratica non si sono ancora sviluppate 
iniziative specifiche in questo senso. Una spiegazione di questo potrebbe trovarsi nel fatto che in ambito civile (enti pubblici governativi, scuole, associazioni, ecc.), si stanno promovendo attività di questa indole e quindi le religioni non vedono questo aspetto così importante- come prioritario. Le entità religiose (specie quelle cristiane) sono di solito più preoccupate dell'azione sociale, nella difesa dei diritti delle persone e di aiuto ai più poveri ed emarginati.

Questa informazione è disponibile sul sito Internet dell'Osservatorio su Sostenibilità e Religioni (sustainabilityandreligions.org) in modo che possa essere a disposizione degli studiosi e dal pubblico generale interessato a questa tematica.

\section{Conclusioni ed implicazioni}

\subsection{Rendere più visibili le attività delle religioni che contribuiscono alla sostenibilità sociale}

Le religioni apparentemente si occupano dell'Aldilà, e quindi potrebbe sembrare che non contribuiscono direttamente allo sviluppo della società o al meno che solo lo fanno a livello teorico o di principi etici. Dai risultati dell'esplorazione quantitativa e qualitativa si vede invece che esiste non solo una preoccupazione per promuovere lo sviluppo, ma che molte di esse realizzano attività che contribuiscono in modo efficace e tangibile alla sostenibilità sociale. Ci sono molte attività promosse nell'ambito della tutela dei diritti umani, della coesione sociale, dell'eguaglianza, della pace, ecc. In molti casi le entità religiose prestano servizi sociali che sono imprescindibili per aiutare le persone in rischio di povertà estrema e di esclusione sociale. Anzi, molte di queste persone si fidano di più dell'aiuto di queste entità che di qualsiasi altra (entità pubbliche e associazioni non religiose). Nella Catalogna la forte crisi economica degli anni 2008-2014 avrebbe avuto conseguenze molto più gravi sui poveri senza il contributo di alcune istituzioni religiose come ad esempio Caritas.

\subsection{La sostenibilità come emergenza etica}

Nei vertici internazionali sullo sviluppo sostenibile si è reso palese il fatto che la sola tecnologia non basta a risolvere i problemi dell'ambiente. Per proporre un esempio, la Conferenza Internazionale dell'ONU sullo sviluppo sostenibile Rio +20 , celebratasi nel 2102 (ONU, 2012) ha constatato un accordo generale sul fatto che i problemi della sostenibilità hanno una chiara dimensione etica. Per far fronte alla crisi globale in cui ci troviamo occorre un consenso basato in valori etici condivisi. La cura del pianeta è un compito per tutti che deve nascere da un impegno personale profondo basato su principi etici. Gli insegnamenti e le credenze di molte delle religioni più numerose nel mondo sono una base valida per trovare questi principi per promuovere comportamenti più sostenibili.

Gli orientamenti etici presenti nel cristianesimo ed in altre confessioni sono capaci di armonizzare la coesione sociale e la pace con la cura e la conservazione delle risorse naturali. Un esempio molto chiaro e recente è l'enciclica Laudato si' di Papa Franceso, nella quale si propone una "sostenibilità integrale" e auspica una "conversione ecologica" non solo individuale ma anche delle intere comunità a livello locale, nazionale ed internazionale (LS, n. 154). Le religioni creazioniste ammettono un universo creato e 
affidato da Dio all'uomo. Da questo principio è possibile apportare ragioni fondate e proposte per curare la creazione e vivere in un modo più equilibrato. Papa Francesco ha fatto chiaro riferimento a questo nel discorso all'Assemblea Generale dell'ONU celebratasi con motivo del Vertice mondiale per l'adozione dell'Agenda 2030 per lo Sviluppo Sostenibile.

L'Osservatorio su Sostenibilità e Religioni pretende anche mostrare il contributo realizzato dalle religioni a livello di riflessione etica, capace di promuovere nelle persone concrete e nelle istituzioni comportamenti più sostenibili.

\subsection{La sostenibilità come base di dialogo interreligioso}

Lo studio che ha dato luogo all'Osservatorio su Sostenibilità e Religioni ha servito anche per evidenziare il fatto che ci siano ampie convergenze tra le religioni nelle tre dimensioni della sostenibilità (sociale e culturale, economica e ambientale). Questi punti in comune potrebbero diventare la base per un proficuo dialogo interreligioso e contribuire specificamente alla pace sociale. La Dichiarazione Congiunta sottoscritta nel 2015 da 154 leader religiosi appartenenti a confessioni diverse e consegnata alla Segreteria Esecutiva della Convenzione delle Nazioni Unite sul Cambiamento Climatico è un esempio di condivisione sui principi basilari delle nostre società e d'impegno delle religioni per il bene comune.

\section{Ringraziamenti}

L'avvio dell'Osservatorio su Sostenibilità e Religioni è stato reso possibile dal finanziamento ottenuto dal Governo autonomo della Catalogna nella convocatoria competitiva RELIG 2014. Ringraziamo per questo la Direcció General d'Afers Religiosos de la Generalitat de Catalunya. Siamo a loro grati anche per averci fornito il banco dati sul quale lavorare (file "Entitats Religioses"). Ringraziamo in secondo termine il gruppo di ricerca diretto dal Dr. A. Belzuncegui, dell'Universitat Rovira i Virgili, il quale ci ha fornito l'elenco di entità cattoliche che realizzano attività sociale nella Catalogna. Per la selezione degli intervistati e per la realizzazione delle interviste è stata decisiva la collaborazione del GTER (Gruppo Stabile di Lavoro delle Religioni), ente creatosi nel 2004. Lo GTER raduna rappresentanti di tutte le confessioni religiose e li coordina e rappresenta davanti i pubblici poteri e la società. L'esperienza dello GTER è unica nella Spagna ed è un'esempio di lavoro coordinato delle religioni al servizio della società. Nel mese di marzo 2015 è stato firmato un accordo tra l'Universitat Internacional de Catalunya ed i GTER per formalizzare un percorso di ricerca sulle religioni nell'ambito accademico universitario.

\section{Riferimenti bibliografici}

ACT Alliance (2015). Statement of Faith and Spiritual Leaders on the upcoming United Nations Climate Change Conference, COP21.Parigi, Dicembre 2015. Disponibile sul sito:http://actalliance.org/wpcontent/uploads/2015/10/COP21_Statement_englisch2.pdf[consul tato a maggio 2016].

Aguilar, M. \&Coscolla, R. (2014). L’acció social des de les entitatsd'Església. El seupaper en la cohesió social a Catalunya. Barcelona: Claret. 
ARC (1986).The Assisi Declarations: Messages on Humanity \& Nature from Buddhism, Christianity, Hinduism, Islam \& Judaism, Assisi 29 September 1986. World Wildlife Fund, 1986. Disponibile sul sito: http://www.arcworld.org/about_ARC.asp[consultato a maggio 2016].

Berry, E. (2014). "Religion and Sustainability in Global Civil Society. Some basic findings from Rio+20".Worldviews: Global Religions, Culture, and Ecology 18.3, 269-288.

Boyd, H. (1999). "Christianity and the Environment in the American Public". Journal for the Scientific Study of Religion, 38, 36-44.

Brown, D. A. (2013). "White paper on the ethical dimensions of climate change". Widener Law School Legal Studies Research Paper, 13-58.

Brundtland, G., et al. (1987). Our Common Future. Report of the World Commission on Environment and Development. Disponibile sul sito: http://www.un-documents.net/our-common-future.pdf [consultato a maggio 2016].

Direcció General d'Afers Religiosos (DGAR), Generalitat de Catalunya. (2014). El mapa de les minoriesreligioses a Catalunya. Disponibilesul sito: http://governacio.gencat.cat/web/.content/afers_religiosos/documents/20150511_informe_map a_religios.pdf [consultato amaggio 2016].

Francesco, Papa (2015a). Incontro con i membri dell'Assemblea Generale dell'Organizzazione delleNazioni Unite, New York, 25-09-2015.Disponibile sul sito:http://w2.vatican.va/content/francesco/it/speeches/2015/september/documents/papafrancesco_20150925_onu-visita.html [consultato a maggio 2016].

Francesco, Papa. (2015b). Lettera Enciclica Laudato Si', sulla cura della casa comune. Vaticano, 25-5-2015. Disponibile sul sito: http://w2.vatican.va/content/francesco/it/encyclicals/documents/papafrancesco_20150524_enciclica-laudato-si.html[consultato a maggio 2016].

González, J. \& Correa, G. (2011). Història del protestantisme a Catalunya (1868-2011), Barcelona: Publicacions del ConsellEvangèlic.

Gottlieb, R. (2006). The Oxford Handbook of Religion and Ecology. New York: Oxford University Press.

Grim, J., \& Tucker, M. E. (2014).Ecology and religion. Washington: Island Press.

Hunt, H. (1999). "Beijing Conversation: Spirituality and Activism". Journal of Feminist Studies in Religion, $15,106-125$.

Interfaith Declaration on Climate Change (IDCC).(2015). Disponibile sul sito:http://www.interfaithdeclaration.org/download/idcc_english.pdf[consultato a maggio 2016].

Intergovernmental Panel on Climate Change (IPCC) (2014).Climate change: Synthesis Report. Disponiblesulsito: http://www.ipcc.ch/pdf/assessment report/ar5/syr/SYR_AR5_FINAL_full.pdf [consultato a maggio 2016].

Manzano, F. (2013). "El Islam y los musulmanes hoy". In A. Orozco de la Torre\& G. Alonso García (a cura di), Dimensión internacional y relaciones con España. Madrid: Escuela Diplomática.

Minton, E. et al. (2015). "Religion and motives for sustainable behaviors: A cross-cultural comparison and contrast". Journal of Business Research 68(9): 1937-1944.

Narayanan, Y. (2013). "Religion and sustainable development: Analysing the connections". Sustainable Development 21(2): 131-139.

Reuter, T. A. (2015). “The Green Revolution in the World's Religions: Indonesian Examples in International Comparison". Religions, 6(4): 1217-1231.

Sherkat, D.E. \& Ellison, Ch.E. (2007). "Structuring the Religion-Enviroment Connection". Journal for the Scientific Study of Religion 46: 71-86.

Taylor, B. (2011). “Toward a Robust Scientific Investigation of the 'Religion'. Variable in the Quest for Sustainability". Journal for the Study of Religion, Nature and Culture 5: 253-262.

Tucker, M. E. (2008). "World Religions, the Earth Charter, and Sustainability". Worldviews: Global Religions, Culture, and Ecology 12.2: 115-128.

UNESCO, Perrot-Lanaud, M. et al. (2005). UNESCO and Sustainable Development. Available online: http://unesdoc.unesco.org/images/0013/001393/139369e.pdf [consultato a maggio 2016].

ONU(2012).Rio+20 The Future we want. Disponibile sul sito:http://www.uncsd2012.org/content/documents/727The\%20Future\%20We\%20Want\%2019 \%20June \%201230pm.pdf[consultato a maggio 2016]. 
ONU(2015a) La ONU elogia la encíclica del Papa Francisco sobre el cambio climático. Disponibile sul sito: http://www.un.org/sustainabledevelopment/es/2015/06/la-onu-elogia-la-enciclica-del-papafrancisco-sobre-el-cambio-climatico/[consultato a maggio 2016].

ONU (2015b).Transforming Our World: the 2030 Agenda for Sustainable Development. Resolution adopted by the General Assembly on 25 September 2015. Reference.(A/70/L.1). Disponibile sul sito: http://sustainabledevelopment.un.org/post2015/transformingourworld [consultato a maggio 2016].

White, L. (1967). “The historical roots of our ecological crisis”.Science155 (3767): 1203-1207.

Wolf, J. \& Gjerris, M. (2009).“A religious perspective on climate change”. Studia Theologica 63(2): 119-139.

WWF (2012 e 2014). Informe Planeta Vivo. Disponibile sul sito: http://www.wwf.es/noticias/informes_y_publicaciones/ [consultato a maggio 2016]. 